\title{
La vivienda social en la transición socioeconómica de los jóvenes. Una revisión sistemática
}

La vivienda social en la transición socioeconómica de los jóvenes. Una revisión sistemática.

Habitação social na transição socioeconômica de jovens. Uma revisão sistemática.

\section{ARTÍCULO GENERAL}

\author{
Mtra. Arq. Mónica Angélica Medina Ramírez \\ https://orcid.org/0000-0002-2353-8087 \\ arq.monica.medina@gmail.com \\ Universidad César Vallejo, Trujillo - Perú
}

Recibido 07 de Junio 2021 | Arbitrado y aceptado 25 de Agosto 2021 | Publicado en 04 Diciembre 2021

\section{RESUMEN}

El presente estudio tuvo como objetivo principal determinar las características de la vivienda social en la transición socioeconómica de los jóvenes. Una revisión sistemática. En este marco la investigación fue de enfoque cualitativo, de tipo descriptivo y de diseño narrativo. Se uso el análisis documental como técnica de recolección de información, para la búsqueda sistemática en 5 plataformas obteniendo, artículos de revistas indexadas como Scielo, Scopus, Redalyc, Dialnet y ProQuest. Tomando en cuenta publicaciones en español, inglés y portugués de los últimos 5 años, subsistiendo al final 40 artículos cualitativos, concluyendo que la vivienda social contemporánea; es una prioridad pública que se hace a través de las políticas públicas, y que se diferencia por su poder adquisitivo. Por tanto, es una expresión material de la cultura y sintetiza los distintos factores que condicionan la habitabilidad. Esto demuestra que la vivienda social es producto de una necesidad global por demanda de espacios o financiamiento subsidiado que está asociado a la tendencia socioeconómica de los jóvenes en los diferentes niveles de la convivencia social.

Palabras clave: vivienda social, demanda de vivienda, subsidio, convivencia social.

\section{ABSTRACT}

The main objective of this study was to determine the characteristics of social housing in the socioeconomic transition of young people. A systematic review. In this framework, the research was qualitative, descriptive and narrative design. Documentary analysis was used as an information collection technique, for the systematic search on 5 platforms, obtaining articles from indexed journals such as Scielo, Scopus, Redalyc, Dialnet and ProQuest. Taking into account publications in Spanish, English and Portuguese of the last 5 years, subsisting at the end 40 qualitative articles, concluding that contemporary social housing; it is a public priority that is made through public policies, and that is differentiated by its purchasing power. Therefore, it is a material expression of culture and synthesizes the different factors that condition habitability. This shows that social housing is the product of a global need for space demand or subsidized financing that is associated with the socioeconomic trend of young people at different levels of social coexistence.

Keywords: social housing, housing demand, subsidy, social coexistence.

\section{RESUMO}

O principal objetivo deste estudo foi determinar as características da habitação social na transição socioeconômica dos jovens. Uma revisão sistemática. Neste quadro, a pesquisa foi qualitativa, descritiva e de desenho narrativo. Utilizou-se a análise documental como técnica de coleta de informações, para a busca sistemática em 5 plataformas, obtendo artigos de periódicos indexados como Scielo, Scopus, Redalyc, Dialnet e ProQuest. Levando em consideração as publicações em espanhol, inglês e português dos últimos 5 anos, subsistindo ao final 40 artigos qualitativos, concluindo que a habitação social contemporânea; é uma prioridade pública que se faz por meio de políticas públicas, e que se diferencia pelo seu poder aquisitivo. Portanto, é uma expressão material da cultura e sintetiza os diferentes fatores que condicionam a habitabilidade. Isso mostra que a habitação social é produto de uma necessidade global de demanda de espaço ou financiamento subsidiado que está associado à tendência socioeconômica de jovens em diferentes níveis de convivência social.

Palavras-chave: habitação social, demanda habitacional, subsídio, convivência social. 


\section{Introducción}

En el estudio de Ibarra \& Martínez (2020), señala que en México la vivienda social implica consolidar en sus ciudadanos la idea de un medio ambiente social adecuado para el crecimiento de sus miembros en un ambiente social cohesionado, donde los vecinos se comprometen solidariamente.

Sin embargo, la presencia del COVID 19 desde el 2020, ha logrado transgredir las fronteras y afectado seriamente las condiciones sociales en las que se desenvuelve porque ha vulnerado la empobrecida economía de las mayorías poblacionales, lo cual ha disminuido las posibilidades de adquisición de una vivienda, o la imposibilidad de continuar rentando una vivienda digna, como es el caso de los inmigrantes. Lossio (2020).

Aunque esta situación se ha agudizado en estos 2 últimos años, la evolución de una vivienda social en el aporte de Hidalgo, Alvarado, Rodríguez, \& Paulsen (2019), ha presentado alternativas en modelos económicos que han proporcionado una alternativa de adquisición tomando un modelo de producción y reproducción de vivienda subsidiada como es el caso de Chile, que alcanzó su éxito por el déficit de calidad urbana, pero que dejó heridas en diferentes sectores de las clases sociales, porque aunque ofreció un efectivo medio de adquisición y bienestar para todo tipo de personas, se puso a prueba la presencia de diversos servicios públicos como salud y educación, para garantizar un crecimiento poblacional con accesibilidad pero que avizoró un paisaje residencial subsidiado.

Por esta razón, Niembro \& Cavanagh (2019), señalan que el mercado laboral en los que residen dentro de esta zona residencial subsidiada tiene mayores dificultades de inserción laboral y por ello la integración a un espacio social de acuerdo a los niveles socioeconómicos que manejan, cada grupo poblacional encuentra dificultades porque se asoma a su desarrollo costumbres tradicionales o populares que difieren de espacios completamente diferenciados con un paisaje residencial que se desajusta al efecto vecindario que normalmente las personas están acostumbradas.

Sin embargo, esto proporciona elementos de políticas públicas para incidir en una integración urbana plena que no limite el acceso a los servicios sino amplié su grado de aplicación.

La vivienda social está sujeta a condiciones geográficas, económicas y sociales que afectan la demanda de la misma en la población, que en su defecto es joven pero que 
además, posee la insignia de inmigrante porque por condiciones socioeconómicas en el contexto global moviliza a generaciones poblacionales a través del orbe; con estos precedentes de información se considera necesario realizar una revisión sistemática para brindar un aporte importante desde una visión global, regional, y local en un contexto donde es posible plantearse la siguiente interrogante:¿Cuáles son las características de vivienda social en la transición socioeconómica de los jóvenes a partir de investigaciones publicadas del 2017 al 2021 ?

Por último, se planteó un objetivo general que fue revisar la vivienda social en la transición socioeconómica de los jóvenes. Una revisión sistemática. Para los objetivos específicos, se planificó identificar estudios sobre vivienda social en jóvenes, a partir de artículos publicados desde el 2017 al 2021; y finalmente se investiga identificar los factores que afectan la vivienda social a partir de revisiones de artículos publicados del 2017 al 2021.

Las teorías que acompañan a presente investigación son 5, la primera es la teoría de la cohesión social que incorpora, la desigualdad social como un elemento importante para su comprensión. Según Barba (2011) estos indicadores están íntimamente ligados porque asocia la armonía social a las condiciones con la que los individuos se organizan para convivir. Los estratos socioeconómicos de una comunidad pueden determinar el espacio o ambiente de convivencia o vivienda.

De otro lado, el estrato sociocultural puede limitar la homogeneidad de una convivencia social saludable e incluso condicionar la posibilidad de insertarse en el mercado laboral.

Esto en principio puede limitar el significado de vivienda social a una estrategia de equilibrio social para legitimar las clases sociales y diferenciarlas por espacio y ubicación. Esto se puede observar en la sociedades modernas o industrializadas donde las desigualdades sociales son evidentes por el estatus laboral, económica y geográfico. Es el caso de la grandes metrópolis o ciudades con un desarrollo económico creciente; es el caso de Hong Kong, Brasilia, Valparaíso, Buenos Aires, e incluso Lima. Los espacios de vivienda son cada vez más reducidos y los vínculos de cohesión tradicionales se desmitifican y la vivienda social o colectiva, se convierte en una vivienda individualista o consumista. 
Esta situación viene dada, y no ha sido algo calculado, porque las relaciones sociales entre segmentos socioeconómicos son estrechamente utilitarias y lo que media en la construcción de espacios de cohesión social son las necesidades prioritarias. Por eso las instituciones públicas entran para mediar alternativas para dar posibilidades a los menos favorecidos y poder limitar la polarización de las poblaciones., para eso de sede educar o democratizar a la sociedad.

De otro lado, el aporte de Garcia (1991) a traves de la teoría de la ciudad se descompone la historia de la humanidad en elementos antropológicos que construyeron la sociedades antiguas y dan una explicación a los parametros historicos de las poblaciones de diferentes urbes. La vivienda social es un componente de desarrollo sostenible para una sociedad en vías de desarrollo, pero que tiene asidero en las necesidades históricas del hombre y que son parte de su desarrollo. La formulación de una sociedad que convalida el derecho a una vivienda a la conformación de la misma por sus creencias o situacion socioeconómica genera tendencias en su desarrollo urbanístico.

Asimismo, el aporte de la teoría del urbanismo, consolida la idea del levantamiento de edificaciones a partir de la organización constructivo espacial, que puede reorganizar el tejido urbano de las ciudades metrópoli como Lima - Capital. En su conjunto se hace una crítica sobre el crecimiento poblacional y la forma de distribución de los sectores socioeconómicos para darle sostenibilidad a la subsistencia de los centros poblacionales.

En este contexto de ciudad se consolida diferentes concepciones ideológicas, entre ellas tenemos, el aporte teórico Álvarez \& Álvarez (2018) que comparte una visión socialista y avizora la concepción de vivienda social teniendo connotaciones $\mathrm{o}$ significados diversos. Para la sociedad cubana la vivienda social es un derecho de todos los ciudadanos que deben estar a su alcance económico, y con el respectivo subsidio del estado, Sin embargo, aunque existen leyes que posibilitan el otorgamiento de subsidios al ejecutarse han traído experiencias nada satisfactorias porque cada familia es particularmente diferente, porque se estandariza casas de $25 \mathrm{~m}^{2}$, aunque existen familias numerosas

Sin embargo, en esta sociedad contemporánea la organización de los espacios y la percepción de los mismos en función de la utilidad hace que el interes social por brindar un espacio adecuado, sea invadido por los intereses particulares como lo señala Sánchez, Pinheiro da Câmara, Teixeira, Araujo, \& Sánchez (2020) el modelo vivienda de interes social trae diversos procesos patológicos que obligan a su conservacion sistematica, y lo 
que deriva en la disminución de la vida útil. Asimismo, Arroyo (2020) maifiesta una definicion amplia sobre la particpación del estado, para la generacion de un espacio de bajo costo, destinado a varios sectores sociales, interviniendo cooperativas, gremios, etc. Por tanto el estado se convierte en un ente de equidad social, lo cual garantiza facilidad para su adquisición, pero también es un canal para que la entidad privada pueda realizar un trabajo binomial. Ambos deben poseer las herramientas de supervision adecuada para garantizar la seguridad y el equilibrio adecuado de estos espacios.

Asimismo, la teoria de Feng Shui consolidad la idea espiritual y de interacción con el entorno que deben poseer cada edificación, y que genera un aporte importante a la vivienda social, como dicen los expertos: "El Feng Shui nos ayuda a equilibrar nuestro entorno para un correcto flujo de la energía y mejorar nuestra interacción con nuestro alrededor" Mao (2014).

Hoy en día, también estamos muy preocupados por las energías que consumimos, por comparar con los pocos recursos que quedan en el planeta. A un nivel arquitectónico, el flujo de energía física o psicológica es de igual importancia para mejorar nuestra vida. "La exigencia de la construcción ya no se plantea solamente en térmicos tectónicos, sino también en los específicamente ambientales: lo visual, lo acústico y lo climático. La lógica de la forma de la arquitectura viene, por lo tanto, determinada también por los parámetros objetivos del ambiente, los cuales entran ya a formar parte de las propuestas para un nuevo orden arquitectónico." Oriol Bohigas.

El Feng Shui siempre busca, también, estar de acuerdo con la naturaleza en muchos aspectos, además de la estabilidad y le confort térmico y es lo que se debe considerar en el estilo de vivienda social para las sociedades contemporáneas.

\section{Metodología}

\subsection{Tipo y diseño de investigación}

Según Hernández, Fernández, \& Baptista (2014), el presente estudio, usa el enfoque de investigación cualitativa porque es una convergencia de concepciones, teorías y técnicas que permiten observar detalladamente el objeto de estudio en su propio contexto y obtener interpretaciones o concepciones de los fenómenos que acontecen. Desde esta perspectiva la revisión sistemática sobre vivienda social para jóvenes adultos reúne la perspectiva de los artículos científicos que interpretan dicho fenómeno, aproximándonos a sus técnicas de estudio y teorías de planificación. Es por lo que no se 
hace ninguna validación numérica de los resultados, sino se hace una interpretación de la situación o fenómeno estudiado. Díaz (2018).

Asimismo, en la perspectiva de Behar (2008), es una investigación de tipo descriptiva, porque analiza cómo se realiza un fenómeno o hecho de estudio que componen la variable vivienda social para jóvenes. De otro lado para Creswell (2005), está sobre el fundamento de un diseño narrativo porque analiza el objeto de estudio para describirlo y analizarlo en función de los acontecimientos que se realizan en las investigaciones.

\subsection{Muestra y criterios de selección}

Para el diseño de este estudio se usaron artículos de revistas indexadas como Scielo, Scopus, Redalyc, Dialnet y ProQuest. Tomando en cuenta publicaciones en español, inglés y portugués de los últimos 5 años. Se ubicaron 65 artículos y luego de filtrar los criterios de exclusión en los que se descartó los duplicados, los estudios fuera del periodo de selección, los estudios cuantitativos y con información innecesaria se obtuvieron finalmente 40 artículos.

\subsection{Técnicas e instrumentos de recolección de datos}

El estudio utilizó la técnica de análisis documental, que permitió recopilar la información bibliográfica de la variable vivienda social situados como artículos científicos, en el contexto de una demanda de vivienda entre los más jóvenes y la carencia de éstos. Esta herramienta permitirá seleccionar información a partir de un esquema que categoriza la información de estos estudios. Díaz ( 2018). 


\subsection{Procedimiento}

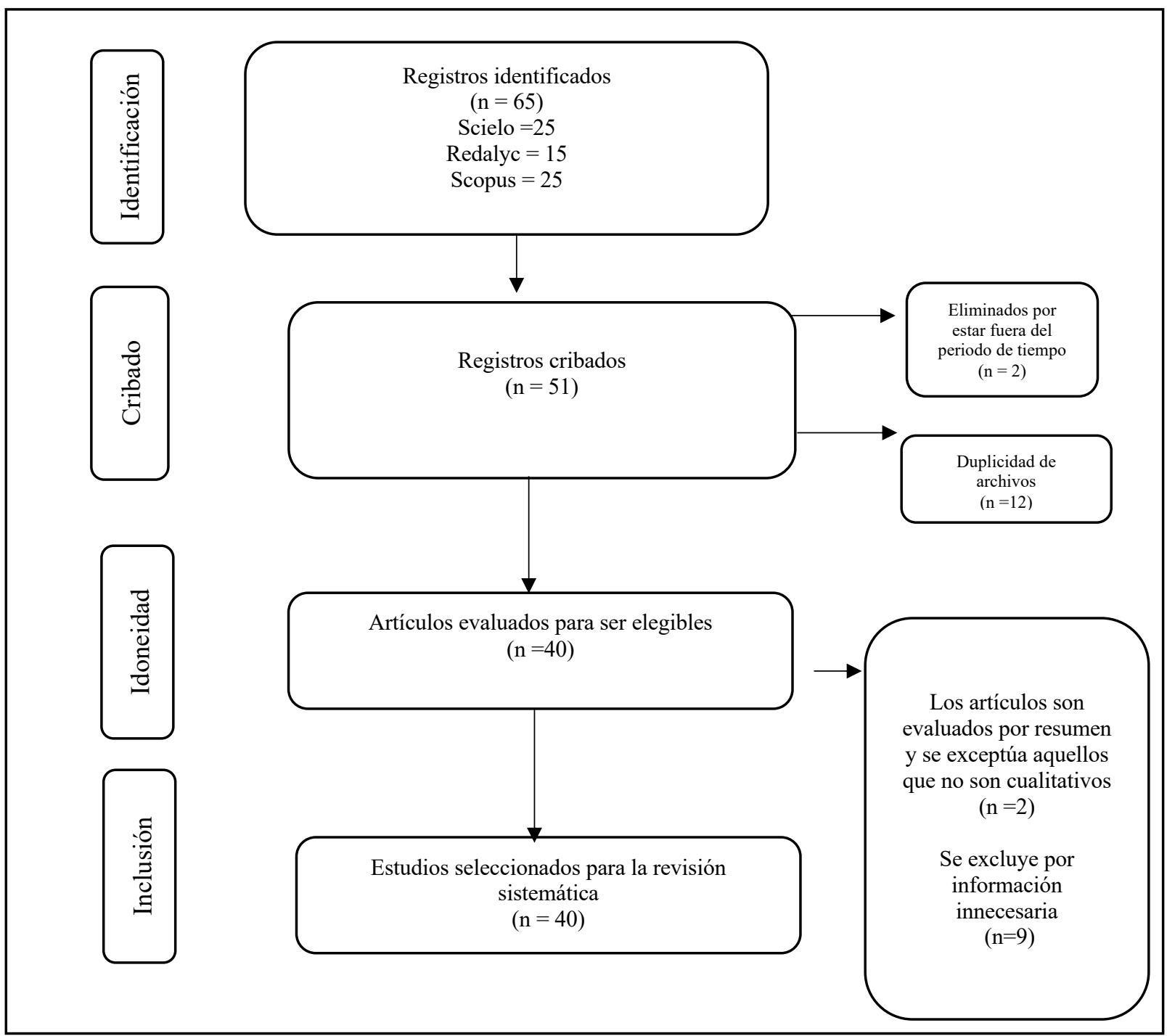

Figura 1. Diagrama Prisma

Elaboración propia. 
El artículo de revisión se inició con la idea de categorizar la vivienda social en la tendencia socioeconómica de los jóvenes, para ello, se realizó la búsqueda en Scielo, Scopus, Redalyc, Dialnet y ProQuest. Los criterios de búsqueda destacables más utilizados para la búsqueda avanzada fueron "vivienda social", "vivienda sostenible", "demanda de vivienda" y "política de vivienda", realizado en Julio del 2021. De lo cual se obtuvo 65 artículos, iniciando un primer filtro descartando aquellos que estén fuera del periodo y estén siendo duplicados, quedando 51 y luego, un segundo filtro que apartó a los estudios que no son cualitativos y aquellos que presentan información innecesaria obteniéndose al final 40 artículos.

\subsection{Método de análisis de la información}

El método de análisis para el procesamiento de la información, se realiza con una base de información para categorizar los principales aportes de cada estudio, para luego analizar, organizar y sintetizar el contenido de cada artículo para presentar las técnicas y resultados de éstos, considerando el enfoque del trabajo de investigación y el objetivo del presente estudio. Hernández, Fernández \& Baptista (2014).

\subsection{Aspectos éticos}

Los aspectos éticos del estudio están en el marco de la integridad científica del documento en elaboración, considerando descartar información falsificada, considerar la autoría de toda información citada, y el respeto íntegro de los resultados de cada investigación consultada, para realizar un análisis concienzudo y respetando las normas internacionales.

\section{Resultados y discusión}

Para responder al objetivo general que fue revisar la vivienda social en la transición socioeconómica de los jóvenes. Una revisión sistemática, se seleccionaron estudios de recolección cualitativa que van desde estudios de caso, análisis biográficos y/o análisis documental de los estudios realizados. Asimismo, la mayoría de estos se encuentran en Latinoamérica, destacando, Argentina, Chile, Cuba, Colombia y Otros países como España e incluso el Perú que localiza la vivienda social en clave a la vivienda autoconstruida porque destacan los aspectos formales y sociales en la arquitectura informal de Lima Metropolitana.

A continuación, se presenta los artículos sobre vivienda social de acuerdo con sus procedimientos o técnicas de recolección de datos: 
Tabla 1: Análisis de las técnicas de recolección de datos e instrumentos utilizados en artículos científicos sobre la temática propia de Investigación.

\begin{tabular}{|c|c|c|c|c|}
\hline Autor & Titulo & Año & Pais & $\begin{array}{l}\text { Procedimeinto de } \\
\text { recoleccion de } \\
\text { datos }\end{array}$ \\
\hline $\begin{array}{l}\text { (Castañeda, } \\
\text { Czajkowski, } \\
\text { \& Gómez, } \\
\text { 2021) }\end{array}$ & $\begin{array}{l}\text { Thermal comfort in } \\
\text { multi-family social } \\
\text { housing in a warm } \\
\text { climate in Colombia. }\end{array}$ & 2021 & Bogota & $\begin{array}{c}\text { Análisis } \\
\text { documental }\end{array}$ \\
\hline $\begin{array}{l}\text { (Domenech, } \\
\text { Gutiérrez, \& } \\
\text { Arauzo } \\
\text { Carod, 2021) }\end{array}$ & $\begin{array}{l}\text { Scenarios post } \\
\text { foreclosure crisis in } \\
\text { Catalonia: accumulation } \\
\text { of housing by banks as } \\
\text { the first step for the rise } \\
\text { of large private } \\
\text { landlords. }\end{array}$ & 2021 & España & Caso de estudio \\
\hline $\begin{array}{l}\text { (Boing \& } \\
\text { Crispim } \\
\text { Boing, 2021) }\end{array}$ & $\begin{array}{l}\text { Inequalities in the access } \\
\text { to healthy urban structure } \\
\text { and housing: an analysis } \\
\text { of the Brazilian census } \\
\text { data. }\end{array}$ & 2021 & Brasil & $\begin{array}{c}\text { Análisis } \\
\text { documental }\end{array}$ \\
\hline $\begin{array}{l}\text { (Ibarra } \\
\text { Salazar \& } \\
\text { Martínez } \\
\text { Ibarra, 2020) }\end{array}$ & $\begin{array}{l}\text { Convivencia vecinal y } \\
\text { satisfacción con la } \\
\text { colonia en los conjuntos } \\
\text { habitacionales de } \\
\text { México. }\end{array}$ & 2020 & México & $\begin{array}{c}\text { Análisis } \\
\text { documental }\end{array}$ \\
\hline
\end{tabular}




\begin{tabular}{|c|c|c|c|c|}
\hline $\begin{array}{l}\text { (Moreira } \\
\text { Carvalho, } \\
2020 \text { ) }\end{array}$ & $\begin{array}{l}\text { Segregação, } \\
\text { vulnerabilidade e } \\
\text { desigualdades sociais e } \\
\text { urbanas. }\end{array}$ & 2020 & Brasil & $\begin{array}{c}\text { Análisis } \\
\text { documental }\end{array}$ \\
\hline $\begin{array}{l}\text { (Arroyo, } \\
2020)\end{array}$ & $\begin{array}{l}\text { Vivienda colectiva y } \\
\text { espacio público. } \\
\text { problemáticas } \\
\text { contemporáneas en } \\
\text { ciudades de Argentina. }\end{array}$ & 2020 & Argentina & $\begin{array}{c}\text { Análisis } \\
\text { documental }\end{array}$ \\
\hline $\begin{array}{l}\text { (Najman, } \\
2020)\end{array}$ & $\begin{array}{l}\text { ¿Relocalizados para vivir } \\
\text { mejor? condiciones de } \\
\text { vida de hogares en un } \\
\text { barrio de vivienda social } \\
1 .\end{array}$ & 2020 & Argentina & $\begin{array}{c}\text { Análisis Biográfico } \\
\text { y/o documental }\end{array}$ \\
\hline
\end{tabular}

(Sánchez

Rodríguez,

Pinheiro da

Câmara de

Queiroz, Manifestaciones

Teixeira patológicas en viviendas $2020 \quad$ Brasil

Pinheiro, de interés social.

Análisis

documental

Araujo

Bertini, \&

Sánchez

García, 2020)

\section{(González}

Vázquez \&

Méndez

Ramírez, 2020)
Efectos de la política

$$
\text { neoliberal en la }
$$

producción de vivienda.

2020 México

Zinacantepec.
Análisis biográfico

y/o documental 
(Rincón

Castellanos,

El derecho a la vivienda.

2020)

Una arquitectura sin piel.

\begin{tabular}{|c|c|c|c|c|}
\hline (Higuera & A referential framework & \multirow{5}{*}{2020} & \multirow{5}{*}{ Mexico } & \multirow{5}{*}{$\begin{array}{c}\text { Análisis } \\
\text { documental }\end{array}$} \\
\hline Zimbrón \& & of social housing: & & & \\
\hline Rivera & International & & & \\
\hline Gutiérrez, & certifications and & & & \\
\hline 2020) & sustainability in Mexico. & & & \\
\hline \multirow{4}{*}{$\begin{array}{l}\text { (Camargo } \\
\text { Sierra, 2020) }\end{array}$} & Vivienda y estrategias & \multirow{4}{*}{2020} & \multirow{4}{*}{ Colombia } & \multirow{4}{*}{$\begin{array}{c}\text { Análisis } \\
\text { documental }\end{array}$} \\
\hline & familiares de vida en & & & \\
\hline & barrios populares & & & \\
\hline & consolidados en Bogotá. & & & \\
\hline
\end{tabular}

\section{El regreso a la ciudad}

(González tradicional. Vivienda

Couret, 2020) social de los 80's en La

$2020 \quad$ Cuba

Análisis

\section{Habana.}

documental

Necropolítica de

(Álvarez de vivienda: 40 años

$\begin{array}{lllll}\text { Andrés, } & \text { desmantelando la } & 2020 & \text { España } & \begin{array}{c}\text { Análisis } \\ \text { documental }\end{array} \\ \text { 2020) } & \text { "informalidad" en } & & & \end{array}$

Madrid (1979-2019).

(Rojas Rubio,

Neumann

Novack,

Habitar el Valparaíso

Vergara neoliberal: vivienda,

Constela, \& hacinamiento y pobreza

Hidalgo como marco de la

Análisis

Dattwyler, pandemia.

2020) 


\begin{tabular}{|c|c|c|c|c|}
\hline $\begin{array}{l}\text { (Giani \& } \\
\text { Türker, 2020) }\end{array}$ & $\begin{array}{l}\text { Assessing flexibility in } \\
\text { real estate mass housing. }\end{array}$ & 2020 & España & $\begin{array}{c}\text { Análisis } \\
\text { documental }\end{array}$ \\
\hline ( Álvarez, & Equipamientos & & & \\
\hline $\begin{array}{l}\text { Ripoll, } \\
\text { Campos, \& }\end{array}$ & $\begin{array}{l}\text { multifuncionales: diez } \\
\text { conjuntos dotacionales }\end{array}$ & 2020 & Argentina & $\begin{array}{c}\text { Análisis } \\
\text { documental }\end{array}$ \\
\hline Ortega, 2020) & en Barcelona. & & & \\
\hline
\end{tabular}

\begin{tabular}{|c|c|c|c|c|}
\hline $\begin{array}{l}\text { (Vergara } \\
\text { d'Alençon, } \\
2020 \text { ) }\end{array}$ & $\begin{array}{l}\text { The role of the civil } \\
\text { society in the } \\
\text { management of housing } \\
\text { and deprived } \\
\text { neighborhoods in Chile. }\end{array}$ & 2020 & Chile & $\begin{array}{c}\text { Análisis } \\
\text { documental }\end{array}$ \\
\hline $\begin{array}{l}\text { (Vieira de, } \\
\text { Denis Granja, } \\
\text { \& Gomes da } \\
\text { Silva, 2020) }\end{array}$ & $\begin{array}{l}\text { Use of a card game tool } \\
\text { to capture end users' } \\
\text { preferences and add } \\
\text { sustainability value to } \\
\text { social housing projects. }\end{array}$ & 2020 & Brasil & $\begin{array}{c}\text { Análisis } \\
\text { documental }\end{array}$ \\
\hline $\begin{array}{l}\text { (Hidalgo } \\
\text { Dattwyler, } \\
\text { Alvarado } \\
\text { Peterson, } \\
\text { Rodríguez } \\
\text { Negrete, \& } \\
\text { Paulsen } \\
\text { Bilbao, 2019) }\end{array}$ & $\begin{array}{l}\text { Paisajes del progreso: } \\
\text { facetas del crecimiento } \\
\text { económico y desarrollo } \\
\text { en las políticas de } \\
\text { vivienda subsidiada en } \\
\text { Santiago y Valparaíso } \\
(1990-2016) .\end{array}$ & 2019 & Chile & Caso de estudio \\
\hline $\begin{array}{l}\text { (Zumelzu \& } \\
\text { Espinoza, } \\
\text { 2019) }\end{array}$ & $\begin{array}{l}\text { Elaboración de una } \\
\text { metodología para evaluar } \\
\text { sostenibilidad en barrios } \\
\text { de ciudades intermedias } \\
\text { en Chile. }\end{array}$ & 2019 & Chile & Caso de estudio \\
\hline
\end{tabular}


(Niembro,

\section{Segregación residencial}

Guevara, \&

socioeconómica e

Cavanagh,

inserción laboral: el caso

de San Carlos de

Análisis

2019)

Bariloche, Argentina.

(Álvarez de

La lucha por la vivienda

André \&

en España (2009-2019):

Smith, 2019)

desde la calle a las

2019 España Caso de estudio

instituciones.

(Matus La integración social

Madrid, como desafío: análisis

Ramoneda, \& del programa de

2019 Chile Caso de estudio

Valenzuela, campamentos en chile

2019) (2011-2018).

(Mercader

Evaluación de impacto

Moyano \&

Camporeale,

ambiental mediante la

2019)

introducción de

2019 España

Caso de estudio

indicadores a un modelo

BIM de vivienda social.

(Gómez

Revisión crítica sobre la

López \&

Castañeda

Nordman,

2019)

construcción de vivienda

estatal y el acceso a

servicios urbanos en el

2019 Argentina

Caso de estudio

área metropolitana de

Tucumán.

Securing the Home:

(Müller, 2019)

Crime, State Sovereignty

and Social Housing in

2019 Medellín

Análisis

Medellín. 
(Rodríguez

Sosa,

Sánchez

Flores, \&

García

Moreno,

Vertical social housing

2019)

\begin{tabular}{|c|c|c|c|c|}
\hline $\begin{array}{l}\text { (Mayorga } \\
\text { Henao, 2019) }\end{array}$ & $\begin{array}{l}\text { Urban facilities: "Places" } \\
\text { of social capital } \\
\text { production. }\end{array}$ & 2019 & Colombia & $\begin{array}{c}\text { Análisis } \\
\text { documental }\end{array}$ \\
\hline
\end{tabular}

Córdoba

Inclusive and resilient

Hernández \& urbanization in informal

García settlements.

Burgos, Exemplification in Latin

2019 Madrid Caso de estudio

2019)

America and the

Caribbean.

El huachafo como clave

de lectura para la

vivienda autoconstruida:

(Dreifuss estudio sobre los

Serrano, aspectos formales y 2019 Perú Caso de estudio

2019) sociales en la

arquitectura informal de

Lima Metropolitana

(Perú).

Operando desde la

(Valderrama forma: un procedimiento

2019 Colombia

Análisis

Vidal, , 2019) para la valoración de la documental vivienda colectiva. 
La vivienda sostenible,

(Valenica, desde un enfoque teórico

2018) y de política pública en

2018 Colombia

Análisis

Colombia.

(Muñoz

Cruz, Arcilla

Bastidas,

López

Meneses,

Aesthetics of social

Delgado

housing: progressive

Echeverri, \& development in Palmira,

2018 Colombia Caso de estudio

Rengifo,

2018)

Colombia (2000-2017).

Gestión de la vivienda a

(Álvarez partir de subsidios.

González \& Barrio La Laguna, Sagua

Álvarez la Grande, Cuba.

2018 Cuba

Análisis

López, 2018) Experiencias de

intervención.

(Álvarez \& Residential conditions

Müller Eie, for immigrant

2018 Noruega

Análisis

2018)

population.

Documental

Towards housing policies

(Lopez \& that consider household's

Paredes, preferences: estimating $2017 \quad$ Chile

2017) the demand for housing

Análisis

attributes in Chile.

(Guzmán

Ramírez \&

Estudio Sobre Las Etapas

De Transformación En 2017 México Caso de estudio

La Vivienda De Interés 


\section{Bravo Patiño, Social. Caso De Estudio: \\ 2017) \\ Fraccionamiento "Valle \\ De Los Naranjos" En La \\ Ciudad De Celaya, \\ Guanajuato.}

(Dávila

Jaramillo \&

Jaramillo

Benavides, 2017)
Arquitectura interior en

viviendas de interés

social: una ruptura de

2017 México

Análisis empírico esquemas.

Diseño de mobiliario en

los primeros

(Gómez multifamiliares de la

Pérez, 2017) Ciudad de México.

2017 México Caso de estudio

Configurar un nuevo

concepto de vivienda.

Al iniciar el análisis de los artículos de investigación se organiza el primer objetivo específico que consiste en identificar los estudios sobre vivienda social para jóvenes, en los artículos revisados desde el año 2017 hasta el año 2021, considerando 5 categorías significativas sobre vivienda social:

La primera, con respecto a la evolución histórica de cohesión en la vivienda social que plantea su transformación, desde González (2020), que profundiza en la historia reciente de los años 80's en materia de vivienda social, en el cual se hace una revalorización de la ciudad tradicional y las tecnologías artesanales de construcción; asimismo, se impulsa la conservación del patrimonio, así como la inserción de nuevos edificios de vivienda en áreas urbanas consolidadas. Asimismo, el aporte de Ibarra \& Martínez (2020), plantea la vivienda social concibiendo en sus ciudadanos la idea de un medio ambiente social que es cohesionado, donde los vecinos se comprometen solidariamente. Sin embargo, para Arroyo (2020), contradice la visión de la vivienda social desde la perspectiva contemporánea, porque el uso de una vivienda colectiva 
tradicional cambia a una concepción individualista, consumista y hedonista, en todos los segmentos sociales, y que ahora esa es la tendencia actual. Esta realidad afecta el concepto de vivienda social porque se diversifica el uso de espacios comunes según las situaciones de uso, esto implica que haya en estos espacios de vivencia colectiva consorcios que mantengan o conserven la utilidad de los espacios. Ya no es una vivencia social subsidiada para atender las necesidades individuales, sino que se convierten en espacios organizados que mantengan las prácticas socioculturales de las áreas comunes.

Segundo, se valida la modalidad de subsidiaridad como alternativa de adquisición de una vivienda social. Así tenemos, la contribución de Hidalgo, Alvarado, Rodríguez, \& Paulsen (2019), que valida la alternativa de adquisición tomando la formula subsidiada como es el caso de Chile, que dejo heridas en diferentes sectores de las clases sociales, porque, aunque ofreció un efectivo medio de adquisición y bienestar para todo tipo de personas, se puso a prueba la presencia de diversos servicios públicos como salud y educación. De otro lado, Niembro \& Cavanagh (2019), señala, que el mercado laboral en los que residen dentro de esta zona residencial subsidiada tiene mayores dificultades de inserción laboral y por ello la integración a un espacio social de acuerdo a los niveles socio económicos que manejan cada grupo poblacional encuentra dificultades porque se asoma a su desarrollo costumbres tradicionales o populares que difieren de espacios completamente diferenciados con un paisaje residencial que se desajusta al efecto vecindario que normalmente las personas están acostumbradas. Por ello, González \& Méndez (2020), hace una reflexión del papel del estado asistencialista y que adquiere el matiz neoliberal para dar más protagonismo a la empresa privada, porque es este último quien marca la lógica del mercado, más que las necesidades de la población, y lo que da libertad a la creación de casquetes urbanos, y un crecimiento sociodemográfico de la periferia por las necesidades de la población.

Tercero, se puede verificar la segregación social en el acceso a la vivienda, lo que se identifica en el estudio de Moreira (2020), que verifica una situación diferenciada para diferentes sectores, tanto para los que viven en el centro de la zona y los que viven en la periferia, afectando el acceso a los bienes, servicios, lo que puede acentuar las vulnerabilidades y las desigualdades. En el caso de Boing \& Boing (2021), se identificó que las familias afroamericanas tenían desigualdades en el acceso a la vivienda, ya sea porque tenían ingresos más bajos, o formación académica insuficiente, teniendo problemas con los servicios básicos, y evidenciándose una escasa infraestructura vial, lo 
mismo podía suceder con los indios americanos o nativos. Encontrándose lugares más desarrollados y con acceso, pero con diferenciaciones raciales. El estudio de Moreira (2020), explica la segregación residencial en Brasil, y tras el análisis documental de los censos del 2000 al 2010, encuentra una situación diferenciada para diferentes sectores, tanto para los que viven en el centro de la zona y los que viven en la periferia, afectando el acceso a los bienes, servicios, lo que puede acentuar las vulnerabilidades y las desigualdades.

Cuarto, el postulado de vivienda social contemporánea; una prioridad publica, que Rincón (2020) señala como una exigencia y un tema de convulsión social, porque las políticas públicas hipotecan la igualdad de los ciudadanos, ya que el que tiene mayor capacidad económica o poder adquisitivo puede adquirir la libertad de un bien como una vivienda, sin embargo, los que no lo tienen, se quedan sin la posibilidad o el derecho de una vivienda digna porque la política neoliberal se ajusta a las reglas de la empresa privada, y el estado subyuga su actuación a sus requerimientos. Sin embargo, se quiere dar una nueva reconceptualización de esta diversificación de la vivienda social. Álvarez de Andrés (2020), viene señalando históricamente que, la ciudad sigue siendo el reflejo de una lucha entre fuerzas de poder desiguales una lucha continua entre prácticas institucionalizadas y de autoorganización, cuyo desenlace viene determinado por el lugar que ocupa una comunidad dentro del espacio social y el modo de vinculación que logra generar. Según Vergara d'Alençon (2020) señala esta cuestión, menos madura en España, se ha venido debatiendo ampliamente en los estudios sobre marginalidad en Latinoamérica, desde la segunda mitad del siglo xx hasta nuestros días, entendiéndose que la superación de la marginalidad implica la transformación del conjunto de la sociedad, una transformación en la que los marginalizados tiene un papel protagónico. Por último, la vivienda social se convierte en una estrategia familiar, como lo señala Camargo (2020), porque se convierte en el seno de una resistencia socioeconómica a los embates de las crisis y puede apalancar por su valor monetario proyectos individuales y/o familiares. Es así como la vivienda social se convierte en una represa para las crisis y un impulso para las propuestas a un estado neoliberal.

Quinto, Álvarez \& Álvarez (2018), señala que la revolución cubana, no ha logrado satisfacer las demandas habitacionales. Las políticas al respecto para el otorgamiento y ejecución de subsidios para casos sociales críticos son insuficientes, Muñoz, Arcilla, López, Delgado \& Rengifo, (2018) en este estudio de caso, explora el desarrollo 
progresivo de proyectos de vivienda social en la ciudad de Palmira (Colombia) entre 2000 y 2017, sugiriendo que las viviendas sociales constituyen un proyecto inacabado, que, moldeado por la subjetividad de sus habitantes, genera conflicto con los espacios públicos. A pesar de su diversidad, las intervenciones siguen patrones identificables, comúnmente vinculados a insuficiencias en los diseños originales.

Para responder al segundo objetivo específico se planteó, identificar los factores que afectan la vivienda social a partir de revisiones de artículos publicados desde el año 2017 hasta el año 2021. Los cuales se expresan en 3 ideas fundamentales:

Primero. - La vivienda social se ve afectada por manifestaciones patológicas como lo manifiesta Higuera \& Rivera (2020), los programas de vivienda desde el año 2000 y hasta el 2018 son una catástrofe. Los complejos habitacionales están plagados de problemas; no hay infraestructura para servicios básicos, agua, drenaje, electricidad. Las calles se hunden, aceras se desmoronan y las plantas de tratamiento de agua no sirven, etc. No obstante, ante esa infinidad de fallas, el principal problema es la corrupción, la impunidad y la indiferencia del gobierno. Las autoridades en turno han sido corresponsables al autorizar complejos habitacionales sin vigilar la aplicación de la norma. Las leyes de zonificación fueron reescritas, plagadas de irregularidades y contubernio con las legislaturas de los estados y se aprobaron los desarrollos sin ninguna supervisión o auditoría.

Segundo. - La vivienda social se afecta por la estructura social, por eso Álvarez \& Müller (2018) señala, que la llegada de grandes poblaciones inmigrantes afecta tanto a la estructura social como urbana de las ciudades de acogida. Los inmigrantes con antecedentes étnicos y sociales similares a menudo ocupan zonas segregadas en las ciudades de acogida, donde las condiciones residenciales son sistemáticamente diferentes de las de otras zonas. Vieira Denis, \& Gomes (2020) explican que, en este sentido, el nivel de integración o segregación espacial es de particular interés. Porque su adaptación es deficiente. Del mismo modo, Dreifuss (2019) señala, que los recientes fenómenos de crecimiento urbano acelerado tienen un impacto muchas veces violento en la imagen de la ciudad. La respuesta de los habitantes frente a la falta de pronta respuesta por parte de las autoridades es automática: son ellos quienes se encargan de dar soluciones a través de la autoconstrucción de una ciudad espontánea, informal, que nace de necesidades cotidianas y recursos limitados. Por medio del estudio de casos en Lima (Perú), Se logra 
trazar una relación entre aspectos formales y ámbitos sociales y culturales, durante la evolución de la vivienda huachafa.

Tercero. - La vivienda social es una estructura de oportunidades desde la percepción de arquitectura interior. En ella según Najman (2020), compara las estructuras de los hogares destinatarios de la vivienda social en dos momentos biográficos: la etapa residencial, inmediatamente anterior a la relocalización, y la etapa residencial actual, considerando en consonancia con los objetivos de la política que dio origen al nuevo Conjunto Urbano. De otro lado, Guzmán \& Bravo (2017) señala, que las etapas de transformación espacial que se presentan en la vivienda de interés social se hacen mediante el análisis de los modos de vida de sus habitantes en reciprocidad con las características arquitectónicas de su vivienda. De otro lado, Rojas, Neumann, Vergara, \& Hidalgo (2020) señala, que la vivienda es una expresión material de la cultura y sintetiza los distintos factores que condicionan su producción y habitabilidad, por esta razón, cualquier cambio en las condiciones del territorio, en la actividad económica de sus habitantes o en sus prácticas sociales, incidirá, tarde que temprano, de forma directa e indirecta, en su expresión formal, material o funcional.

Al sintetizar los resultados encontrados se puede ratificar el postulado de Barba (2011), que asocia la armonía social a las condiciones con la que los individuos se organizan para convivir. Esto coincide con los postulados de González (2020), que revaloriza la ciudad tradicional y las tecnologías artesanales de construcción; para impulsar la conservación del patrimonio. Además, el aporte de Ibarra \& Martínez (2020), plantea la vivienda social concibiendo en sus ciudadanos la idea de un medio ambiente social que es cohesionado, donde los vecinos se comprometen solidariamente. Lo que conlleva a ratificar el principio de homogeneidad de una convivencia social saludable e incluso condicionar la posibilidad de insertarse en el mercado laboral. De otro lado, el teórico García (1991), que valora la vivienda social como un componente de desarrollo sostenible para una sociedad en vías de desarrollo, pero que tiene asidero en las necesidades históricas del hombre y que son parte de su desarrollo. Esto se acopla a la contribución de Hidalgo, Alvarado, Rodríguez, \& Paulsen (2019), que valida la alternativa de adquisición tomando la formula subsidiada poniendo a prueba la presencia de diversos servicios públicos como salud y educación. Por ello, González \& Méndez (2020), hace una reflexión del papel del estado asistencialista y que adquiere el matiz neoliberal para dar más protagonismo a la empresa privada, porque es este último quien 
marca la lógica del mercado, más que las necesidades de la población, y lo que da libertad a la creación de casquetes urbanos, y un crecimiento sociodemográfico de la periferia por las necesidades de la población. Esto se complementa con el aporte de la teoría del urbanismo, porque consolida la idea del levantamiento de edificaciones a partir de la organización constructivo espacial, por ello se hace una crítica sobre el crecimiento poblacional y la forma de distribución de los sectores socioeconómicos para darle sostenibilidad a la subsistencia de los centros poblacionales.

\section{Conclusiones}

1. La vivienda social ha tenido una evolución histórica desde una perspectiva latinoamericana y se consolida la cohesión social como una realidad que afecta el concepto de vivienda social porque ya no solo atiende las necesidades individuales, sino que se convierten en espacios organizados que mantengan las prácticas socioculturales de las áreas comunes.

2. La alternativa de la fórmula subsidiada es diferente según sectores de las clases sociales, y según la zona residencial subsidiada tiene mayores dificultades porque diferencia la integración de acuerdo con los niveles socioeconómicos que manejan cada grupo poblacional y genera un papel del estado asistencialista.

3. Se afecta tanto a la estructura social que los antecedentes étnicos y sociales señalan zonas segregadas en las ciudades, donde las condiciones residenciales son sistemáticamente diferentes de las de otras zonas.

4. La vivienda social contemporánea; es una prioridad pública que se hace a través de las políticas públicas y que la diferencia por el poder adquisitivo. Por tanto, es una expresión material de la cultura y sintetiza los distintos factores que condicionan habitabilidad, Sin embargo, se quiere dar una nueva reconceptualización de esta diversificación de la vivienda social.

\section{Recomendaciones}

1. Se recomienda un estudio local en el contexto histórico de la vivienda social por regiones del país. Diagramando las principales características socioculturales y económicas y las necesidades individuales de los ciudadanos. 
2. Analizar fórmulas económicas alternas para aplicar al acceso de una vivienda social desde los sectores socioeconómicos más empobrecidos y de acuerdo con la población económicamente activa con mayor capacidad de pago y de expansión.

3. Estudiar los casos más significativos de vivienda social en ciudades desarrolladas como Hong Kong o Brasilia. Creando un modelo de proyecto de vivienda social acorde a la estructura de residencia cultural de su localidad.

4. Considerar nuevos diseños tecnológicos con sostenimiento ambiental para corresponder a una vivienda social contemporánea que tenga prioridad en la cultura y la conservación del medio ambiente.

\section{Referencias bibliográficas}

Álvarez de André, E., \& Smith, H. (2019). La lucha por la vivienda en España (20092019): desde la calle a las instituciones. Revista invi.

Álvarez de Andrés, E. (2020). Necropolítica de vivienda: 40 años desmantelando la “informalidad” en Madrid (1979-2019).

Álvarez González, C. E., \& Álvarez López, A. E. (2018). Gestión de la vivienda a partir de subsidios. Barrio La Laguna, Sagua la Grande, Cuba. Experiencias de intervención. Arquitectura y Urbanismo.

Álvarez, A. L., \& Müller Eie, D. (2018). Residential conditions for immigrant population.

Álvarez, A., Ripoll, V., Campos, L., \& Ortega, A. (2020). Guidelines for BIM implementation in the environmental assessment of social housing. Revista Esoa.

Arroyo, J. (2020). Vivienda colectiva y espacio público. problemáticas contemporáneas en ciudades de Argentina. Revista Legado de Arquitectura y Diseño.

Behar Rivero, D. S. (2008). Metodología de la investigación.

Boing, A. F., \& Crispim Boing, A. (2021). Inequalities in the access to healthy urban structure and housing: an analysis of the Brazilian census data.

Camargo Sierra, A. P. (2020). Vivienda y estrategias familiares de vida en barrios populares consolidados en Bogotá. Revista INVI.

Castañeda, W., Czajkowski, J. D., \& Gómez, A. F. (2021). Thermal comfort in multifamily social housing in a warm climate in Colombia. 
Cerino. (2021). Jornadas laborales prolongadas causan 745 mil muertes al año. según un estudio. Obtenido de https://notipress.mx/vida/jornadas-laborales-prolongadas745-mil-muertes-estudio-7399

Córdoba Hernández, R., \& García Burgos, A. P. (2019). Inclusive and resilient urbanization in informal settlements. Exemplification in Latin America and the Caribbean. Bitácora Urbano Territorial, 30.

Creswell, J. (2005). Educational research: Planning, conducting, and evaluating quantitative and qualitative research. Pearson Education.

Dávila Jaramillo, M. E., \& Jaramillo Benavides , A. S. (2017). Arquitectura interior en viviendas de interés social: una ruptura de esquemas. Revista Arquitectura y Urbanismo.

Diaz Herrera, C. (2018). Investigación cualitativa y análisis de contenido temático. Orientación intelectual de revista Universum.

Domenech, A., Gutiérrez, A., \& Arauzo Carod, J. M. (2021). Scenarios post foreclosure crisis in catalonia: accumulation of housing by banks as the first step for the rise of large private landlords. Revista Erkunde.

Dreifuss Serrano, C. (2019). El huachafo como clave de lectura para la vivienda autoconstruida: estudio sobre los aspectos formales y sociales en la arquitectura informal de Lima Metropolitana (Perú). Arquitectura Revista.

Giani, G., \& Türker, Ö. O. (2020). Asessing flexibility in real estate mass housing. Arquitectura Revista.

Gómez López, C. F., \& Castañeda Nordman, A. L. (2019). Revisión crítica sobre la construcción de vivienda estatal y el acceso a servicios urbanos en el área metropolitana de Tucumán. Revista de Arquitectura y Urbanismo.

Gómez Pérez, T. L. (2017). Diseño de mobiliario en los primeros multifamiliares de la Ciudad de México. Configurar un nuevo concepto de vivienda. Revista Legado de Arquitectura y Diseño.

González Couret, D. (2020). El regreso a la ciudad tradicional. Vivienda social de los 80’s en La Habana. Revista INVI.

González Vázquez, S., \& Méndez Ramírez, J. J. (2020). Efectos de la política neoliberal en la producción de vivienda. Zinacantepec. Arquitectura y Urbanismo. 
Guzmán Ramírez, A., \& Bravo Patiño, M. A. (2017). Estudio Sobre Las Etapas De Transformación En La Vivienda De Interés Social. Caso De Estudio: Fraccionamiento "Valle De Los Naranjos" En La Ciudad De Celaya, Guanajuato. Revista Legado de Arquitectura y Diseño.

Hernández Sampieri, R., Fernández Collado, C., \& Baptista Lucio, M. (2014). Metodología de la investigación (6ta edcion ed.). México DF.

Hidalgo Dattwyler, R., Alvarado Peterson, V., Rodríguez Negrete, L., \& Paulsen Bilbao, A. (2019). Paisajes del progreso: facetas del crecimiento económico y desarrollo en las políticas de vivienda subsidiada en Santiago y Valparaíso (1990-2016).

Higuera Zimbrón, A., \& Rivera Gutiérrez, E. (2020). A referential framework of social housing: International certifications and sustainability in México. Revista de Estudios Territoriales.

Ibarra Salazar, J., \& Martínez Ibarra, A. (2020). Convivencia vecinal y satisfacción con la colonia en los conjuntos habitacionales de México. Revista Mexicana de Ciencias Políticas y Sociales.

Lopez, E., \& Paredes, D. (2017). Towards housing policies that consider household's preferences: estimating the demand for housing attributes in Chile. International Journal of Strategic Property Management.

Lossio, J. (2020). Covid-19 en el Perú: respuestas estatales y sociales.

Matus Madrid, C. P., Ramoneda, Á., \& Valenzuela, F. (2019). La integración social como desafío: análisis del programa de campamentos en chile (2011-2018). Revista invi.

Mayorga Henao, J. M. (2019). Urban facilities: "Places" of social capital production. Revista de Arquitectura.

Mercader Moyano, M., \& Camporeale, P. E. (2019). Evaluación de impacto ambiental mediante la introducción de indicadores a un modelo BIM de vivienda social. Revista Hábitat Sustentable.

Moreira Carvalho, I. (2020). Segregação, vulnerabilidades e desigualdades sociais e urbanas. Revista de ciencias sociales.

Müller, F. (2019). Securing the Home: Crime, State Sovereignty and Social Housing in Medellín. Revista Europea de Estudios Latinoamericanos y del Caribe. 
Muñoz Cruz, L. M., Arcilla Bastidas, J. P., López Meneses, I. S., Delgado Echeverri, J. J., \& Rengifo, R. A. (2018). Aesthetics of social housing: progressive development in Palmira, Colombia (2000-2017). Revista INVI.

Najman, M. (2020). ¿Relocalizados para vivir mejor? condiciones de vida de hogares en un barrio de vivienda social 1. Revista de Arquitectura y Urbanismo.

Niembro, A., Guevara, T., \& Cavanagh, E. (2019). Segregación residencial socioeconómica e inserción laboral: el caso de san carlos de Bariloche, Argentina. Revista invi.

OMS. (2020). Garantizar la seguridad de los trabajadores de la salud para preservar la de los pacientes. Obtenido de https://www.who.int/es/news/item/17-09-2020-keephealth-workers-safe-to-keep-patients-safe-who

Rincón Castellanos, M. (2020). El derecho a la vivienda. Una arquitectura sin piel. Geopauta.

Rodríguez Sosa, M., Sánchez Flores, E., \& García Moreno, G. (2019). Vertical social housing on the northern border of Mexico: criteria for sustainable densification. Revista INVI.

Rojas Rubio, I., Neumann Novack, P., Vergara Constela, C., \& Hidalgo Dattwyler, R. (2020). Habitar el Valparaíso neoliberal: vivienda, hacinamiento y pobreza como marco de la pandemia. O Social em Questão,.

Sánchez Rodríguez, F., Pinheiro da Câmara de Queiroz, L. A., Teixeira Pinheiro, L., Araujo Bertini, A., \& Sánchez García, F. (2020). Manifestaciones patológicas en viviendas de interés social. Revista Arquitectura y Urbanismo.

Valderrama Vidal, , J. C. (2019). Operando desde la forma: un procedimiento para la valoración de la vivienda colectiva. Revista de Arquitectura.

Valenica, D. E. (2018). La vivienda sostenible, desde un enfoque teórico y de política pública en Colombia. Revista Ingenierías Universidad de Medellín.

Vergara d'Alençon, L. M. (2020). The role of the civil society in the management of housing and deprived neighbourhoods in Chile. Revista INVI.

Vieira de, A., Denis Granja, A., \& Gomes da Silva, V. (2020). Use of a card game tool to capture end users' preferences and add sustainability value to social housing projects. Revista Ambiente Construido. 
Zumelzu, A., \& Espinoza, D. (2019). Elaboración de una metodología para evaluar sostenibilidad en barrios de ciudades intermedias en Chile. Revista 180. 